KYUNGPOOK Math. J. 55(2015), 817-826

http://dx.doi.org/10.5666/KMJ.2015.55.4.817

pISSN 1225-6951 eISSN 0454-8124

(C) Kyungpook Mathematical Journal

\title{
Some Analogues of a Result of Vasconcelos
}

\author{
DAvid EARL DobBs* \\ Department of Mathematics, University of Tennessee, Knoxville, Tennessee 37996- \\ 1320, USA \\ e-mail : dobbs@math.utk.edu \\ JAY Allen Shapiro \\ Department of Mathematics, George Mason University, Fairfax, Virginia 22030- \\ 4444, USA \\ e-mail : jshapiro@gmu.edu
}

Abstract. Let $R$ be a commutative ring with total quotient ring $K$. Each monomorphic $R$-module endomorphism of a cyclic $R$-module is an isomorphism if and only if $R$ has Krull dimension 0. Each monomorphic $R$-module endomorphism of $R$ is an isomorphism if and only if $R=K$. We say that $R$ has property $(\star)$ if for each nonzero element $a \in R$, each monomorphic $R$-module endomorphism of $R / R a$ is an isomorphism. If $R$ has property $(\star)$, then each nonzero principal prime ideal of $R$ is a maximal ideal, but the converse is false, even for integral domains of Krull dimension 2. An integral domain $R$ has property ( $\star$ ) if and only if $R$ has no $R$-sequence of length 2 ; the "if " assertion fails in general for non-domain rings $R$. Each treed domain has property $(\star)$, but the converse is false.

\section{Introduction}

All rings considered in this note are commutative; all rings and modules are unital. Our starting point is the following result of Vasconcelos [14, Theorem]: a ring $R$ has (Krull) dimension 0 if and only if each monomorphic $R$-module endomorphism of a finitely generated $R$-module is surjective (that is, is an isomorphism). While

* Corresponding Author.

Received June 21, 2013; accepted September 12, 2013.

2010 Mathematics Subject Classification: Primary 13A15, 13C99; Secondary 13G05, 13F05, 20M14, 13B30.

Key words and phrases: Commutative ring, cyclic module, monomorphism, Krull dimension, monoid ring, integral domain, pullback, treed domain, pseudo-valuation domain, total quotient ring, localization.

The second-named author thanks the University of Tennessee for its warm hospitality during his visit in 2013. 
the statement of this result concerns module-theoretic behavior, its proof involves a central ring-theoretic result of Cohen-Seidenberg (cf. [4]), namely, that the partners of any integral ring extension must have the same dimension. In considering whether a result like that of Vasconcelos could be proved without recourse to such ringtheoretic tools, we found an elementary module-theoretic proof of the following analogue (see Proposition 2.1): a ring $R$ has (Krull) dimension 0 if and only if each monomorphic $R$-module endomorphism of a cyclic $R$-module is an isomorphism. The rest of this note examines what can be said about a ring $R$ if one imposes such a condition on only some of its cyclic modules.

For instance, Proposition 2.2 shows each monomorphic $R$-module endomorphism of (the cyclic $R$-module) $R$ is an isomorphism if and only if $R$ is a total quotient ring. This is perhaps of greater interest because total quotient rings can be of arbitrary dimension (cf. [12]), but one would seek greater generality if $R$ is a (commutative integral) domain, since domains which are total quotient rings must be fields (and hence of no ideal-theoretic interest). For this reason, we focus on rings $R$ that have the following property, which we call property $(\star)$ : for each nonzero element $a \in R$, each monomorphic $R$-module endomorphism of (the cyclic $R$-module) $R / R a$ is an isomorphism.

It is shown in Proposition 2.4 that rings having property $(\star)$ retain some of the low-dimensional flavor of the rings studied by Vasconcelos, in the sense that whenever $R$ has property $(\star)$, each nonzero principal prime ideal of $R$ is a maximal ideal. However, Example 2.6 shows that the converse is false, even for two-dimensional domains. A very useful result for domains is given in Proposition 2.5, where it is shown that a domain $R$ has property $(\star)$ if and only if there is no $R$-sequence on $R$ of length 2. Examples of domains of arbitrary dimension that have property $(\star)$ are provided by Corollary 2.9: if $R$ is a pseudo-valuation domain (in the sense of [11]), then $R$ has property $(\star)$. In addition, Example 2.6 is shown to be best-possible by Corollary 2.8, where it is shown that each one-dimensional domain has property $(\star)$. More generally, Corollary 2.7 establishes that each treed domain has property $(\star)$. Additional examples of domains having property $(\star)$ are given in Theorem 2.10, which provides some pullbacks, not all of which are treed domains, that have property $(\star$ ). Remark 2.12 (a) shows that the converse of Corollary 2.7 is false, while Remark 2.12 (d) shows that the criterion in Corollary 2.5 involving $R$-sequences does not characterize property $(\star)$ for non-domains. Finally, Remark 2.12 (e) shows that property $(\star)$ is somewhat more fragile than zero-dimensionality in regard to its possible stability under globalization.

As usual, if $A$ is a ring, it will be convenient to denote the (Krull) dimension of $A$ by $\operatorname{dim}(A)$; the set of units of $A$ by $U(A)$; the set of prime ideals of $A$ by $\operatorname{Spec}(A)$; the radical of an ideal $I$ of $A$ by $\sqrt{I}$; the set of zero-divisors of $A$ by $Z(A)$; and the total quotient ring of $A$ by $\operatorname{tq}(A):=A_{A \backslash Z(A)}$. Also, if $E$ is an $A$-module and $P \in \operatorname{Spec}(A)$, we let $E_{P}$ denote the localization of $E$ at $A \backslash P$. Parts (d) and (e) of Remark 2.12 use the idealization construction of Nagata; a useful reference for basic facts about idealization is [12]. Any unexplained material is standard, as in the textbooks listed in the bibliography. 


\section{Results}

We begin in Proposition 2.1 by showing how a focus on cyclic modules can lead to a simple analogue of the motivating result of Vasconcelos.

Proposition 2.1. Let $R$ be a ring. Then the following conditions are equivalent:

(1) If $E$ is a cyclic $R$-module and $f \in \operatorname{Hom}_{R}(E, E)$ is an injection, then $f$ is an isomorphism;

(2) $\operatorname{dim}(R)=0$.

Proof. $(1) \Rightarrow(2)$ : The contrapositive of this implication can be proved as in the proof of $[14$, Theorem]. For the sake of completeness, we next provide the details. If (2) fails, pick distinct prime ideals $P \subset Q$ of $R$, pick an element $b \in Q \backslash P$, put $E:=R / P$, let $f$ be the element of $\operatorname{Hom}_{R}(E, E)$ defined by multiplication by $b$, and note that $f$ is an injection but not a surjection, so that (1) fails.

$(2) \Rightarrow(1)$ : Suppose that $\operatorname{dim}(R)=0$. Let $E$ be a cyclic $R$-module and suppose that $f \in \operatorname{Hom}_{R}(E, E)$ is an injection. We will show that $f$ is surjective. By hypothesis, there exists an ideal $I$ of $R$ such that $E \cong R / I$. Of course, $f$ is surjective (respectively, injective) if and only if the induced map $f_{M} \in \operatorname{Hom}_{R_{M}}\left(E_{M}, E_{M}\right)$ is surjective (respectively, injective) for each $M \in \operatorname{Spec}(R)$; and for any such $M$, we have $E_{M} \cong R_{M} / I R_{M}$, which is a cyclic $R_{M}$-module. Thus, without loss of generality, we may take $(R, M)$ quasi-local and $E=R / I$. Also without loss of generality, $I \neq R$ (since the only endomorphism of the module 0 is surjective), and so $I \subseteq M$. In fact, $\sqrt{I}=M$ by [9, Corollary 2.10], since the zero-dimensionality of $R$ implies that $M$ is the unique prime ideal of $R$. Next, note that $f(1+I)=b+I$ for some $b \in R$. (If $I \neq 0$, then $b$ is not uniquely determined, but it suffices to pick any such $b$.) Then $f$ is simply multiplication by $b$; that is, if $r \in R$, then $f(r+I)=b r+I$ $(=b \cdot(r+I))$. If $b \in U(R)$, then $f\left(b^{-1}+I\right)=b b^{-1}+I=1+I$, and so $f$ is surjective since $f$ is $R$-linear. Hence, without loss of generality, $b \in R \backslash U(R)=M$. It suffices to obtain a contradiction in this case. As above, we have that $\sqrt{R b}=M$, and so $b \in \sqrt{I}$. Hence, there is a smallest positive integer $n$ such that $b^{n} \in I$. Consider $\rho:=b^{n-1} \in R$. Then $\rho+I \neq 0 \in E$, although $f(\rho+I)=b \rho+I=b^{n}+I=$ $0 \in E$, which contradicts the assumption that $f$ is a monomorphism. The proof is complete.

We next consider rings that satisfy various weaker versions of condition (1) of Proposition 2.1. By focusing on just the cyclic $R$-module $R$, Proposition 2.2 gives a new characterization of total quotient rings. As is well known (cf. [12, Theorems 26.2 and 26.4]), such rings can be of arbitrary Krull dimension.

Proposition 2.2. Let $R$ be a ring. Then the following conditions are equivalent:

(1) If $f \in \operatorname{Hom}_{R}(R, R)$ is an injection, then $f$ is an isomorphism;

(2) $R=\operatorname{tq}(R)$.

Proof. The result holds for the zero ring (as its total quotient ring must sensibly be defined to also be the zero ring). Thus, without loss of generality, $R \neq 0$. If $b \in R$, 
let $\tilde{b} \in \operatorname{Hom}_{R}(R, R)$ denote multiplication by $b$, namely, the map given by $r \mapsto b r$. As $b$ varies over the elements of $R, \tilde{b}$ varies over the elements of $\operatorname{Hom}_{R}(R, R)$. Moreover (since $R \neq 0$ ), $f=\tilde{b}$ is a monomorphism if and only if $b \notin Z(R)$. On the other hand, $f=\tilde{b}$ is surjective if and only if $b \in U(R)$ (in which case, $f=\tilde{b}$ is clearly an isomorphism). Thus, (1) holds if and only if $R \backslash Z(R)=U(R)$. Since it is clear that (2) holds if and only if $R \backslash Z(R)=U(R)$, the proof is complete.

Seeking a condition that is, in a sense, midway between condition (1) of Proposition 2.1 and condition (1) of Proposition 2.2, we next introduce the main object of study in this note. We will say that a ring $R$ has property $(\star)$ if, for each nonzero element $a \in R$, each monomorphic $R$-module endomorphism of (the cyclic $R$-module) $R / R a$ is an isomorphism. Our first examples of such rings are given in Corollary 2.3, which is an immediate consequence of Proposition 2.1 (or of Vasconcelos' motivating result [14, Theorem]).

Corollary 2.3. Each ring of Krull dimension 0 has property $(\star)$.

We next show that any ring $R$ that has property $(\star)$ must exhibit certain behavior that was established in [6, Corollary 2.4] for any going-down domain, namely, that each nonzero principal prime ideal of $R$ is a maximal ideal.

Proposition 2.4. Let $R$ be a ring that has property $(\star)$. Then each nonzero principal prime ideal of $R$ is a maximal ideal.

Proof. It suffices to rework the proof of the implication (1) $\Rightarrow(2)$ in Proposition 2.1 (taking $P$ in that proof to be the given nonzero principal prime ideal and $Q$ to be, if possible, a strictly larger prime ideal of $R$ ).

Example 2.6 will show that the converse of Proposition 2.4 is false. First, it is convenient to note that for domains, property $(\star)$ admits the following reformulation involving $R$-sequences (also known as regular sequences).

Proposition 2.5. Let $R$ be a domain. Then $R$ has property $(\star)$ if and only if there is no $R$-sequence (on $R$ ) of the form $a, b$.

Proof. For all $a, b \in R$, let $\widetilde{(a, b)}$ denote the $R$-module homomorphism $\widetilde{(a, b)}$ : $R / R a \rightarrow R / R a$ given by $r+R a \mapsto b r+R a$, for each $r \in R$. Note that $R$ fails to have property $(\star)$ if and only if there exist elements $a, b$ in $R$ such that $a \neq 0$ and $\widetilde{(a, b)}$ is injective but not surjective. On the other hand (cf. [13, page 84]), if $a$ and $b$ are elements of $R$, then $a, b$ is an $R$-sequence (on $R$ ) if and only if $R a+R b \neq R$, $a \neq 0$ and $\widetilde{(a, b)}$ is injective.

Suppose first that the "if" assertion fails. Hence, $R$ has no $R$-sequences consisting of two elements, but there exist elements $a, b$ in $R$ such that $a \neq 0$ and $\widetilde{(a, b)}$ is injective but not surjective. Consequently $R a+R b=R$, and so $R / R a=R(b+R a)=\widetilde{(a, b)}(R / R a)$, whence $\widetilde{(a, b)}$ is surjective, the desired contradiction.

Next, suppose that the "only if" assertion fails. Then $R$ has an $R$-sequence $a, b$, but $R$ has property ( $\star$ ). The $R$-sequence condition gives us that $R a+R b \neq R$, 
$a \neq 0$ and $\widetilde{(a, b)}$ is injective. But then property $(\star)$ ensures that $\widetilde{(a, b)}$ is surjective. Thus $R / R a=\widetilde{(a, b)}(R / R a)=(R a+R b) / R a$, and so a standard homomorphism theorem yields that $R=R a+R b$, the desired contradiction, which completes the proof.

Recall from [3] that a domain is said to be an antimatter domain if it has no irreducible elements. Note that any antimatter domain $D$ vacuously satisfies the conclusion of Proposition 2.4; Example 2.6 will show that such $D$ need not satisfy the hypothesis of Proposition 2.4. Also, it will be convenient to let $\mathbb{Q}^{+}$denote the monoid consisting of the non-negative rational numbers under addition.

Example 2.6. Let $K$ be a perfect field of positive characteristic, let the monoid $S$ be the cardinal sum of two copies of $\mathbb{Q}^{+}$, and let $R$ denote the monoid ring $K[X ; S]$. Then $R$ is a domain of Krull dimension 2 and each nonzero principal prime ideal of $R$ is a maximal ideal, but the ring $R$ does not have property ( $\star$ ).

Proof. By [1, Theorem 5 (1)], $R$ is an antimatter domain (and a GCD-domain). By combining [10, Theorems 21.4 and 17.1] with [9, Theorem 30.5], we see that the dimension of this domain is the torsion-free rank of $S$, namely, 2. Since $R$ is an antimatter domain, it has no nonzero principal prime ideals (and so, vacuously, any such ideal is maximal). It remains only to show that $R$ does not have property $(\star)$. By Proposition 2.5, it therefore suffices to produce an $R$-sequence $a, b$ (on $R$ ). The reader can easily check that $a:=X^{(0,1)}, b:=X^{(1,0)}$ form the desired $R$-sequence. $\square$

Recall that a domain $R$ is said to be treed if $\operatorname{Spec}(R)$, as a poset under inclusion, forms a tree; that is, if no prime ideal of $R$ can contain incomparable prime ideals of $R$. Corollary 2.7 will lead to many examples of rings that have property $(\star)$ by showing that any treed domain has property $(\star)$. In this vein, and with an eye to the comment that preceded Proposition 2.4, we recall that any going-down domain is a treed domain [5, Theorem 2.2] (and the converse is false).

Corollary 2.7. If $R$ is a treed domain, then $R$ has property $(\star)$.

Proof. By [7, Theorem 2.2], there is no $R$-sequence (on $R$ ) of the form $a, b$. Therefore, an application of Proposition 2.5 completes the proof.

By Corollary 2.7 and the comment preceding it, each going-down domain has property $(\star)$. The applications in the next two corollaries could be inferred from this fact about going-down domains, but we prefer to present those corollaries as consequences of Corollary 2.7, as treed domains are perhaps more widely known than going-down domains.

Corollary 2.8. If $R$ is a domain of Krull dimension at most 1 , then $R$ has property $(\star)$.

Proof. Since $R$ is a treed domain, the assertion follows from Corollary 2.7. For an alternate proof, combine Proposition 2.5 with the well-known fact (cf. [13, Exercise 
22 , page 104]) that for any non-negative integer $n$, an $n$-dimensional ring cannot contain an $R$-sequence of length $n+1$.

In view of Corollary 2.8 and Example 2.6, the following result is of some interest, as pseudo-valuation domains can have arbitrary Krull dimension. (To see this, either use [6, Lemma 4.5 (v), Proposition 4.9 (i)] or consider a valuation domain whose value group has rank equal to the desired dimension.)

Corollary 2.9. Each pseudo-valuation domain has property $(\star)$.

Proof. Each pseudo-valuation domain is a treed domain [11, Corollary 1.3]. Therefore, an application of Corollary 2.7 completes the proof.

The conclusion in Corollary 2.9 applies as well, and more generally, to the class of locally pseudo-valuation domains (the so-called LPVDs). Since the property of being treed is a local property of domains, this raises the question whether property $(\star)$ itself is a local property. We will address some related issues in Remark 2.12. First, we give a family of examples of rings having property $(\star)$ in Theorem 2.10. As explained in Remark 2.12(c), some of those rings are not treed.

Theorem 2.10. Let $(T, M)$ be a quasi-local domain with property $(\star)$, let $D$ be a subring of $T / M$, let $\pi: T \rightarrow T / M$ denote the canonical projection map, and let $R$ be the pullback $R:=D \times_{T / M} T\left(=\pi^{-1}(D)\right)$. Then $R$ has property $(\star)$ if and only if $D$ has property $(\star)$.

Proof. We have that $R / M \cong D$, and so $M \in \operatorname{Spec}(R)$. Also, by the order-theoretic impact of the topological description of $\operatorname{Spec}(R)$ (with the Zariski topology) in [8, Theorem 1.4], each prime ideal of $R$ is comparable with $M$ under inclusion, and the set of prime ideals of $R$ that contain (resp., are contained in) $M$ is isomorphic as a poset to $\operatorname{Spec}(D)$ (resp., $\operatorname{Spec}(T))$.

We will prove the "if" assertion first. So, suppose that $D$ has property $(\star)$. By Proposition 2.5, it suffices to derive a contradiction from the assumption that $a, b$ is an $R$-sequence on $R$. Our analysis will break naturally into two cases that are determined by where $a$ and $b$ reside relative to $M$. In any event, note that $0 \neq a, b \in R \backslash U(R)$; and that $b, a$ is also an $R$-sequence on $R$, by [7, Lemma 2.1 (b)].

Case 1: either $a \notin M$ or $b \notin M$. By the preceding observation, we may assume, without loss of generality, that $a \notin M$. Now, by a standard homomorphism theorem, $D / D a \cong R /(R a+M)$ as $R$-modules. It will simplify the notation to point out that $R a+M=R a$; that is, $M \subseteq R a$. (In detail, note that $a \in T \backslash M=U(T)$, so that, working in $T$, we have $M=\left(a^{-1} M\right) a \subseteq(T M) a=M a \subseteq R a$.) Let $f$ denote multiplication by $b$ on $D / D a$. We claim that $f$ is a monomorphism. Suppose, on the contrary, that there exists $t \in R \backslash R a$ such that $b t \in R a$. Since $a, b$ is an $R$ sequence on $R$, we conclude that $t \in R a$, the desired contradiction. This proves the above claim; that is, $f$ is a monomorphism.

Since $D$ has property $(\star)$ and $f$ is a monomorphism, $f$ must be surjective. 
Therefore, there exists $u \in R$ such that $b u-1 \in R a+M=R a$. Thus, $1 \in R a+R b$, contradicting that $a, b$ is an $R$-sequence on $R$.

Case 2: both $a$ and $b$ are elements of $M$. Since $a, b$ is an $R$-sequence on $R$, we have that $b \notin R a$. We claim that $b \notin T a$. To see this, suppose, on the contrary, that $b=q a$ for some $q \in T \backslash R$. Then $q \in U(T)$ (since $T \backslash U(T)=M \subseteq R$ ). Observe that $b \in M \backslash R a$, and so $b+R a \neq 0 \in R / R a$. However, $b \cdot(b+R a)=b^{2}+R a=$ $\left(q^{2} a\right) a+R a=0 \in R / R a$, since $q^{2} a \in T a \subseteq M \subseteq R$. This contradicts that $a, b$ is an $R$-sequence on $R$, thus proving the above claim; that is, $b \notin T a$.

Note that multiplication by $b$ on $T / T a$ is not surjective, since the image of this endomorphism is contained in $M / T a$. Consequently, since $T$ has property $(\star)$, this endomorphism is not injective. Hence, there exists $c \in T \backslash T a$ such that $b c \in T a$. Write $b c=t a$, with $t \in T$. If $c \in U(T)$, then $b^{2}=\left(t c^{-1} b\right) a \in R a$ (since $t c^{-1} b \in T b \subseteq M \subseteq R$ ), so that multiplication by $b$ annihilates the nonzero element $b+R a \in R / R a$, which contradicts that $a, b$ is an $R$-sequence on $R$. Therefore, $c \in T \backslash U(T)=M \subseteq R$. As $b c=t a$ and $c \notin R a$, the fact that $a, b$ is an $R$ sequence on $R$ ensures that $t \notin R$. In particular, $t \notin M$, and so $t^{-1} \in T$. Then $d:=c t^{-1}$ satisfies $b d=a$ and $d \in M T=M \subseteq R$. As $a, b$ is an $R$-sequence on $R$, it follows that $d \in R a$. Thus, $c=t d \in T(R a)=T a$, the desired contradiction. This completes the proof of the "if" assertion.

Suppose the "only if" assertion fails. Then $R$ has property $(\star)$ but $D$ does not have property $(\star)$. Use $\left.\pi\right|_{R}$ to identify $D=R / M$. By Proposition 2.5 , there exist $a, b \in R$, such that $\bar{a}:=a+M, \bar{b}:=b+M$ is a regular sequence on $D$ (that is, an " $R$ "-sequence of $D$-modules). Necessarily, neither $a$ nor $b$ is an element of $M$. As above, a standard homomorphism theorem gives that $D / D \bar{a} \cong R /(R a+M)$. By Proposition 2.5, it cannot be the case that $a, b$ is an $R$-sequence on $R$. It also cannot be the case that $R a+R b=R$ (since $D \bar{a}+D \bar{b} \neq D$ ). Hence, there exists $s \in R \backslash R a$ such that $b s \in R a$. Then, a fortiori, $b s \in R a+M$, whence $\bar{s}:=s+M$ satisfies $\bar{b} \cdot \bar{s} \in D \bar{a}$. Since $\bar{a}, \bar{b}$ is a regular sequence on $D$, it follows that $\bar{s} \in D \bar{a}$; that is, $s \in R a+M$. However, $M=\left(M a^{-1}\right) a \subseteq(M T) a=M a \subseteq R a$, and so $s \in R a$, the desired contradiction, which completes the proof.

Corollary 2.11. Let $R \subseteq T$ be domains such that $\operatorname{Spec}(R)=\operatorname{Spec}(T)$ as sets. If $T$ has property $(\star)$, then $R$ has property $(\star)$.

Proof. As any field has property $(\star)$, we may assume that $R$ and $T$ are not fields. Also without loss of generality, $R \neq T$. Then, by [2, Proposition 3.3 and Theorem 3.25], $R$ is a quasi-local ring, say with maximal ideal $M$, such that if $K$ denotes the quotient field of $R$ and $\left(M:_{K} M\right):=\{u \in K \mid u M \subseteq M\}$, with $\pi:\left(M:_{K}\right.$ $M) \rightarrow\left(M::_{K} M\right) / M$ the canonical projection map, then there exists a field $k \subseteq$ $\left(M:_{K} M\right) / M$ such that $T$ equals the pullback $\pi^{-1}(k)$. As $T$ is quasi-local and $\bar{k}$ has property $(\star)$, the assertion now follows from Theorem 2.10. For an alternate proof, one could combine [7, Proposition 2.5] with the criterion for property $(\star)$ in Proposition 2.5.

Since any pseudo-valuation domain can be obtained as the pullback of its canonically associated valuation domain and a subfield of the residue field of that valuation 
domain (cf. [2, Proposition 2.6]), Corollary 2.11 reduces the assertion in Corollary 2.9 to the case of a valuation domain. An additional application of Theorem 2.10 will be given in Remark 2.12 (c).

Remark 2.12. (a) The converse of Corollary 2.7 is false, even for two-dimensional Noetherian domains. Indeed, let $R$ be any two-dimensional Noetherian local domain which is not a Cohen-Macaulay ring. An example of such $R$ was given in $[7$, Remark $2.4]$, where it was also shown (for any such $R$ ) that $R$ has no $R$-sequence of the form $a, b$. Hence, by Proposition $2.5, R$ has property $(\star)$. Of course, $R$ is not treed (cf. [13, Theorem 144]).

(b) Many examples of valuation domains that are also antimatter domains were given in [3]. By Corollary 2.7 (or Corollary 2.9), each of those domains has property $(\star)$. Their behavior is to be contrasted with that of the antimatter domain given in Example 2.6. Noetherian domains form another prominent class of domains that contains some domains having property $(\star)$ and some domains that do not have property $(\star)$. Indeed, recall from (a) that any two-dimensional (Noetherian) local non-Cohen-Macaulay domain has property $(\star)$, while one can show, by combining Proposition 2.4 with [13, Theorems 5 and 142], that any Noetherian unique factorization domain of Krull dimension at least 2 must fail to have property $(\star)$. These facts help to show the diversity of the class of domains that have property $(\star)$. Our point here is that if $D$ is a Noetherian domain (more generally, a domain satisfying the ascending chain condition on principal ideals) but not a field, then $D$ is far from being an antimatter domain, since each nonzero nonunit of $D$ is a product of irreducible elements of $D$.

(c) Consider the data in Theorem 2.10, with $D$ also assumed to be quasi-local and treed. Once again, we use the order-theoretic impact of the topological description of $\operatorname{Spec}(R)$ (with the Zariski topology) in [8, Theorem 1.4]. This shows that $R$ is quasi-local and also that $R$ is treed if and only if $T$ is treed; in addition, it follows from [8, Proposition 2.1 (5)] that $\operatorname{dim}(R)=\operatorname{dim}(D)+\operatorname{dim}(T)$. Thus, if $T$ is one of the two-dimensional Noetherian local non-Cohen-Macaulay domains considered in (a) and $D$ is a $d$-dimensional valuation subring of the field $T / M$, then Theorem 2.10 implies that the pullback $R:=D \times_{T / M} T$ is a $(d+2)$-dimensional non-treed domain having property $(\star)$, where, as usual, we take $\infty+2:=\infty$.

(d) Proposition 2.5 does not extend to arbitrary rings. In fact, the "if" assertion of Proposition 2.5 fails for the following data. Consider the ring $A:=\mathbb{Z}_{2 \mathbb{Z}}$ and the idealization $R:=A(+) A$. Then $R$ is a non-domain (since $Z(R)=0(+) A \neq 0$ ); $R$ is a Noetherian local one-dimensional ring with unique maximal ideal $2 A(+) A$; and Proposition 2.4 shows that $R$ does not have property $(\star)$, since $0(+) A=A(0,1)$ is a nonzero principal prime ideal of $R$ which is not a maximal ideal of $R$. Finally, since $\operatorname{dim}(R)<2$, it follows from [13, Theorem 132] that $R$ has no $R$-sequence of the form $a, b$.

(e) We consider here the behavior of property $(\star)$ under globalization. In this paragraph, we get a positive result for domains. To wit: if $R$ is a domain such that $R_{M}$ has property $(\star)$ for each maximal ideal $M$ of $R$, then $R$ has property $(\star)$. For 
a proof, we may assume, without loss of generality, that $R$ is not a field. Then the assertion follows by combining [7, Lemma 2.1 (a)] with the criterion for property ( $\star$ in Proposition 2.5.

Next, if the ambient ring $R$ is not necessarily a domain, we have the following positive result. If $R$ is a ring whose Jacobson radical $J(R)$ contains $Z(R)$ and if $R_{M}$ has property ( $\star$ ) for each maximal ideal $M$ of $R$, then $R$ has property $(\star)$. For a proof, let $0 \neq a \in R$ and consider an arbitrary monomorphism $f \in \operatorname{Hom}_{R}(R / R a, R / R a)$. Our task is to show that $f$ is an isomorphism or, equivalently, that the induced map $f_{M}:(R / R a)_{M} \rightarrow(R / R a)_{M}$ is an isomorphism for each maximal ideal $M$ of $R$. Of course, $f_{M}$ inherits the property of being a monomorphism from $f$, for each $M$. Note also that for each $M$, we have a canonical isomorphism $(R / R a)_{M} \cong R_{M} /(R a)_{M}$ and $(R a)_{M}=R_{M}(a / 1)$. Therefore, since each $R_{M}$ is assumed to have property $(\star)$, we will be done if $(a / 1) \neq(0 / 1) \in R_{M}$ for each $M$. This, in turn, follows from the hypothesis that $Z(R) \subseteq J(R)$ (for if $(a / 1) \neq(0 / 1)$ in $R_{M}$, then $\left(0:_{R} a\right) \nsubseteq M$, although $\left(0:_{R} a\right) \subseteq Z(R) \subseteq J(R) \subseteq M$, the desired contradiction).

We pause to give an example of a quasi-local non-domain $R$ satisfying the hypothesis that $Z(R) \subseteq J(R)$ from the preceding paragraph. Let $A$ and $R=A(+) A$ be as constructed in $(\mathrm{d})$. Then $Z(R)=0(+) A \subset 2 A(+) A=J(R)$. Notice that we showed in (d) that $R$ does not have property $(\star)$.

Finally, despite the positive results in the first two paragraph of this part (e), we show next that property $(\star)$ does not globalize in general. In other words, there exists a ring $R$ such that $R_{M}$ has property $(\star)$ for each maximal ideal $M$ of $R$ but $R$ does not have property $(\star)$. Necessarily, by the above remarks, any such $R$ must fail to be a domain and must fail to satisfy that $Z(R) \subseteq J(R)$. To construct a satisfactory $R$, we let $(A, N)$ be a quasi-local treed domain which is not a field, and put $R:=A \times A$. The maximal ideals of $R$ are $N \times A$ and $A \times N$; the localizations of $R$ at these maximal ideals are each canonically isomorphic as rings to $A_{N} \cong A$. In particular, both of these localizations are treed domains and so, by Corollary 2.7, $R_{M}$ has property $(\star)$ for each maximal ideal $M$ of $R$. It remains to show that $R$ does not have property $(\star)$. To that end, first use the hypothesis that $A$ is not a field to pick a nonzero nonunit $\rho \in N$, and then let $a:=(1,0), b:=(0, \rho) \in R$. It suffices to show that if $f$ denotes multiplication by $b$ on $E:=R / R a$, then $f$ is a monomorphism but not an isomorphism. By means of the canonical isomorphism $E \cong A, f$ can be identified with the $A$-module homomorphism $A \rightarrow A$ given by multiplication by $\rho$. This map is clearly a monomorphism but not surjective.

In closing, we raise the following question about localization which is, in a sense, dual to the question that was studied in Remark 2.12(d). Can one find useful conditions on a ring $R$ that has property $(\star)$ and a multiplicatively closed subset $S$ of $R$ so as to ensure that $R_{S}$ also has property $(\star)$ ? 


\section{References}

[1] D. D. Anderson, J. Coykendall, L. Hill and M. Zafrullah, Monoid domain constructions of antimatter domains, Comm. Algebra, 35(2007), 3236-3241.

[2] D. F. Anderson and D. E. Dobbs, Pairs of rings with the same prime ideals, Canad. Math. J., 2(1980), 363-384.

[3] J. Coykendall, D. E. Dobbs and B. Mullins, On integral domains with no atoms, Comm. Algebra, 27(1999), 5813-5831.

[4] I. S. Cohen and A. Seidenberg, Prime ideals and integral dependence, Bull. Amer. Math. Soc., 52(1946), 252-261.

[5] D. E. Dobbs, On going-down for simple overrings, II, Comm. Algebra, 1(1974), 439458.

[6] D. E. Dobbs, Coherence, ascent of going-down and pseudo-valuation domains, Houston J. Math., 4(1978), 551-567.

[7] D. E. Dobbs, Treed domains have grade 1, Internat. J. Commut. Rings, 2(2003), $43-46$.

[8] M. Fontana, Topologically defined classes of commutative rings, Ann. Mat. Pura Appl., 123(1980), 331-355.

[9] R. Gilmer, Multiplicative Ideal Theory, Dekker, New York, 1972.

[10] R. Gilmer, Commutative Semigroup Rings, Univ. Chicago Press, Chicago/London, 1984.

[11] J. R. Hedstrom and E. G. Houston, Pseudo-valuation domains, Pacific J. Math., 75(1978), 137-147.

[12] J. A. Huckaba, Commutative Rings with Zero Divisors, Dekker, New York, 1988.

[13] I. Kaplansky, Commutative Rings, rev. ed., Univ. Chicago Press, Chicago/London, 1974.

[14] W. V. Vasconcelos, Injective endomorphisms of finitley generated modules, Proc. Amer. Math. Soc., 25(1970), 900-901. 